\title{
Frequency-domain ray series for viscoelastic waves with a non-symmetric stiffness matrix
}

\author{
LUDĚK KLIMEŠ
}

Department of Geophysics, Faculty of Mathematics and Physics, Charles University, Ke Karlovu 3, 12116 Praha 2, Czech Republic (http://sw3d.cz/staff/klimes.htm)

Received: September 8, 2017; Revised: November 29, 2017; Accepted: December 14, 2017

\begin{abstract}
In an elastic medium, it was proved that the stiffness tensor is symmetric with respect to the exchange of the first pair of indices and the second pair of indices, but the proof does not apply to a viscoelastic medium. In order to indicate which phenomena could be observed in the wave field if the stiffness matrix were nonsymmetric, we propose the frequency-domain ray series for viscoelastic waves with a non-symmetric stiffness tensor in this paper.
\end{abstract}

Keywords: viscoelastic media, stiffness tensor, wave propagation, ray theory, ray series, travel time, amplitude

\section{INTRODUCTION}

The $3 \times 3 \times 3 \times 3$ complex-valued frequency-domain stiffness tensor (elastic tensor, tensor of elastic moduli) $c^{i j k l}=c^{i j k l}\left(x^{m}, \omega\right)$, projecting the strain tensor onto the stress tensor, depends on spatial coordinates $x^{m}$ and circular frequency $\omega$. It is symmetric with respect to the first pair of indices

$$
c^{i j k l}=c^{j i k l}
$$

and with respect to the second pair of indices

$$
c^{i j k l}=c^{i j l k} \text {. }
$$

It is thus frequently expressed in the form of the $6 \times 6$ stiffness matrix whose lines correspond to the first pair of indices and columns to the second pair of indices according to the Voigt notation.

In an elastic medium, it was proved that the stiffness tensor is symmetric with respect to the exchange of the first pair of indices and the second pair of indices,

$$
c^{i j k l}=c^{k l i j}
$$

The $6 \times 6$ stiffness matrix is thus symmetric in an elastic medium. The ray theory for a $3-\mathrm{D}$ smoothly heterogeneous anisotropic elastic medium with a symmetric 


\section{Kliměs}

stiffness matrix is well developed (Babich, 1961; Červený, 1972; 2001; Chapman, 2004; Červený et al., 2007).

However, the above mentioned proof does not apply to a viscoelastic medium, and we do not know whether symmetry (3) holds in a viscoelastic medium. It is then reasonable to indicate which phenomena could be observed in the wave field if the stiffness matrix were non-symmetric. Analogously to Thomson (1997), we thus do not assume symmetry (3) in this paper, and propose the frequency-domain ray series for viscoelastic waves with a non-symmetric stiffness matrix,

$$
c^{i j k l} \neq c^{k l i j} \text {. }
$$

The lower-case Roman indices take values 1, 2 and 3. The Einstein summation over repetitive lower-case Roman indices is used throughout the paper.

\section{STANDARD RAY SERIES}

In the frequency domain, the viscoelastodynamic equation for complex-valued displacement $u_{i}=u_{i}\left(x^{m}, \omega\right)$ reads

$$
\left(c^{i j k l} u_{l, k}\right)_{, j}-(\mathrm{i} \omega)^{2} \varrho u_{i}=0 \quad,
$$

where lower-case Roman subscript,$k$ following a comma denotes the partial derivative with respect to the corresponding spatial coordinate $x^{k}$. Here $c^{i j k l}=c^{i j k l}\left(x^{m}, \omega\right)$ is the complex-valued frequency-domain stiffness tensor, $\varrho=\varrho\left(x^{m}\right)$ is the density and $\omega$ is the circular frequency.

We express the displacement in terms of its complex-valued vectorial amplitude $U_{i}=U_{i}\left(x^{m}, \omega\right)$ and complex-valued travel time $\tau=\tau\left(x^{m}\right)$ as

$$
u_{i}=U_{i} \exp (\mathrm{i} \omega \tau) \quad .
$$

We expand the frequency-dependent amplitude into high-frequency asymptotic series

$$
U_{i}=\sum_{n=0}^{\infty}(\mathrm{i} \omega)^{-n} U_{i}^{[n]},
$$

where $U_{i}^{[n]}=U_{i}^{[n]}\left(x^{m}, \omega\right)$ is the $n$-th order vectorial amplitude.

We insert displacement (6) into viscoelastodynamic equation (5) and obtain equation

$$
(\mathrm{i} \omega)^{2} N^{i}\left(U_{m}, \tau_{, u}\right)+\mathrm{i} \omega M^{i}\left(U_{m}, \tau_{, u}\right)+L^{i}\left(U_{m}\right)=0,
$$

where the differential operators are defined as

$$
\begin{gathered}
N^{i}\left(U_{m}, \tau_{, u}\right)=\varrho\left[\Gamma^{i l}\left(x^{m}, \tau_{, u}\right) U_{l}-U_{i}\right], \\
M^{i}\left(U_{m}, \tau_{, u}\right)=\left(c^{i j k l} \tau_{, k} U_{l}\right)_{, j}+c^{i j k l} \tau_{, j} U_{l, k},
\end{gathered}
$$

and

$$
L^{i}\left(U_{m}\right)=\left(c^{i j k l} U_{l, k}\right)_{, j} \quad .
$$

Here the Christoffel matrix, defined as

$$
\Gamma^{i l}\left(x^{m}, p_{u}\right)=a^{i j k l}\left(x^{m}\right) p_{j} p_{k} \quad,
$$


is a function of six phase-space coordinates $x^{m}, p_{u}$ formed by three spatial coordinates $x^{m}$ and three slowness-vector components $p_{u}$ which represent the spatial gradient of travel time $\tau, p_{u}=\tau_{, u}$. In definition (12),

$$
a^{i j k l}\left(x^{m}\right)=c^{i j k l}\left(x^{m}\right)\left[\varrho\left(x^{u}\right)\right]^{-1}
$$

is the density-reduced stiffness tensor.

In order to obtain the standard ray series, we insert series (7) into viscoelastodynamic equation (8) and sort the terms according to the order of $i \omega$, analogously to Babich (1961) or Cervený (1972; 2001, Sec. 5.7). We then obtain the system of equations

$$
N^{i}\left(U_{k}^{[n]}, \tau_{, l}\right)+M^{i}\left(U_{k}^{[n-1]}, \tau_{, l}\right)+L^{i}\left(U_{k}^{[n-2]}\right)=0
$$

for each order $n=0,1,2, \ldots$. Here $U_{k}^{[-1]}=0$ and $U_{k}^{[-2]}=0$, i.e. operator $M^{i}$ is missing in this equation for $n=0$ and operator $L^{i}$ is missing in this equation for $n=0,1$.

Note that the system of equations (14) does not follow necessarily from viscoelastodynamic equation (8). The above sorting according to the order of $i \omega$ is our assumption leading to the standard ray series. If we wish to obtain another kind of ray series, e.g., the coupling ray series, we may choose a solution of viscoelastodynamic equation (8) different from the system of equations (14), see Klimeš (2013).

\section{CHRISTOFFEL EQUATION AND EIKONAL EQUATION}

Equation (14) for $n=0$ constitutes the matrix Christoffel equation

$$
\Gamma^{i l}\left(x^{m}, \tau_{, u}\right) U_{l}^{[0]}-U_{i}^{[0]}=0 .
$$

The three eigenvalues of Christoffel matrix (12) correspond to three waves: the $\mathrm{P}$ wave and two $\mathrm{S}$ waves. Unlike as in the elastic case, the Christoffel matrix is not symmetric.

We select one of three eigenvalues of Christoffel matrix (12) and denote it as $G=$ $G\left(x^{m}, \tau_{, u}\right)$. We denote the corresponding right-hand eigenvector of the Christoffel matrix as $g_{i}=g_{i}\left(x^{m}, \tau_{, u}\right)$,

$$
\Gamma^{i l} g_{l}=G g_{i},
$$

and the corresponding left-hand eigenvector of the Christoffel matrix as $\vec{g}_{i}=$ $\vec{g}_{i}\left(x^{m}, \tau, u\right)$,

$$
\vec{g}_{i} \Gamma^{i l}=\vec{g}_{l} G
$$

The three right-hand eigenvectors of the Christoffel matrix and the three left-hand eigenvectors of the Christoffel matrix are mutually biorthogonal.

The lengths of the three right-hand eigenvectors of the Christoffel matrix are not determined, but we choose the lengths of the corresponding left-hand eigenvectors so that the three right-hand eigenvectors and the three left-hand eigenvectors are mutually biorthonormal,

$$
\vec{g}_{k} g_{k}=1
$$




\section{Kliměs}

The zero-order vectorial amplitude then reads

$$
U_{i}^{[0]}=U^{[0]} g_{i},
$$

where the zero-order ray-theory amplitude $U^{[0]}$ will be determined by the transport equation in Section 6.

Whereas the two $\mathrm{S}$ waves, which propagate with different velocities, are linearly polarized in elastic media, they may be elliptically or even circularly polarized in viscoelastic media. Whereas the two elliptically polarized $\mathrm{S}$ waves always display equal handedness for a symmetric stiffness matrix, they display opposite handedness for a sufficiently non-symmetric stiffness matrix.

In order to satisfy Christoffel equation (15), selected eigenvalue $G$ must be unit,

$$
G\left(x^{m}, \tau_{, u}\right)=1
$$

Nonlinear first-order partial differential equation (20) for travel time $\tau$ is called the Hamilton-Jacobi equation. In wave propagation problems, it is also often referred to as the eikonal equation. The methods for solving the Hamilton-Jacobi equation are already mostly developed (Hamilton, 1837; Červený, 1972; Klimeš, 2002; 2010; 2016).

Hamilton-Jacobi equation (20) generates the equations of rays (Hamilton equations, equations of geodesics) and the related equations such as the Hamiltonian equations of geodesic deviation (dynamic ray tracing equations).

The equations of rays may be expressed in various forms, e.g., as

$$
\begin{gathered}
\frac{\mathrm{d} x^{i}}{\mathrm{~d} \gamma}=\frac{1}{2} \frac{\partial G}{\partial p_{i}}, \\
\frac{\mathrm{d} p_{i}}{\mathrm{~d} \gamma}=-\frac{1}{2} \frac{\partial G}{\partial x^{i}},
\end{gathered}
$$

where parameter $\gamma$ along rays coincides with the values of travel time $\tau$. Refer to Section 4 for the case of complex-valued eigenvalue $G=G\left(x^{m}, p_{u}\right)$ and parameter $\gamma$.

Hereinafter, $\frac{\partial G}{\partial x^{i}}$ and $\frac{\partial G}{\partial p_{i}}$ denote the partial derivatives of function $G\left(x^{m}, p_{u}\right)$ of six phase-space coordinates $x^{m}, p_{u}$. Using this notation, the partial derivatives of any function $G\left(x^{m}, \tau_{, u}\left(x^{a}\right)\right)$ of three spatial coordinates read

$$
G_{, j}=\frac{\partial G}{\partial x^{j}}+\frac{\partial G}{\partial p_{k}} \tau_{, k j}
$$

Since

$$
G\left(x^{m}, p_{u}\right)=\vec{g}_{r}\left(x^{m}, p_{u}\right) \Gamma^{r s}\left(x^{a}, p_{b}\right) g_{s}\left(x^{c}, p_{d}\right),
$$

the first-order phase-space derivatives of eigenvalue $G\left(x^{m}, p_{u}\right)$ can be expressed as

$$
\frac{\partial G}{\partial p_{i}}\left(x^{m}, p_{u}\right)=\vec{g}_{r}\left(x^{m}, p_{u}\right) \frac{\partial \Gamma^{r s}}{\partial p_{i}}\left(x^{a}, p_{b}\right) g_{s}\left(x^{c}, p_{d}\right)
$$

and

$$
\frac{\partial G}{\partial x^{i}}\left(x^{m}, p_{u}\right)=\vec{g}_{r}\left(x^{m}, p_{u}\right) \frac{\partial \Gamma^{r s}}{\partial x^{i}}\left(x^{a}, p_{b}\right) g_{s}\left(x^{c}, p_{d}\right)
$$


Note that the phase-space derivatives of the left-hand and right-hand eigenvectors in the above derivatives (25)-(26) of relation (24) vanish because of definitions (16)(17) and normalization condition (18).

We insert definition (12) into phase-space derivatives (25)-(26), and obtain expressions

$$
\frac{\partial G}{\partial p_{i}}\left(x^{m}, p_{u}\right)=\vec{g}_{r}\left(x^{m}, p_{u}\right)\left[a^{r i k s}\left(x^{a}\right)+a^{r k i s}\left(x^{a}\right)\right] p_{k} g_{s}\left(x^{c}, p_{d}\right)
$$

and

$$
\frac{\partial G}{\partial x^{i}}\left(x^{m}, p_{u}\right)=\vec{g}_{r}\left(x^{m}, p_{u}\right) \frac{\partial a^{r j k s}}{\partial x^{i}}\left(x^{a}\right) p_{j} p_{k} g_{s}\left(x^{c}, p_{d}\right)
$$

Note that

$$
V^{i}\left(x^{m}, p_{u}\right)=\frac{1}{2} \vec{g}_{r}\left(x^{m}, p_{u}\right)\left[a^{r i k s}\left(x^{a}\right)+a^{r k i s}\left(x^{a}\right)\right] p_{k} g_{s}\left(x^{c}, p_{d}\right)
$$

is the ray-velocity vector, which is sometimes also referred to as the group-velocity vector, e.g., by Červený (2001).

\section{COMPLEX-VALUED AND REAL-VALUED RAYS}

The above relations (20)-(29) are applicable both to real-valued travel time $\tau$ and complex-valued travel time $\tau$. The methods for their solution are obvious if eigenvalue $G=G\left(x^{m}, p_{u}\right)$ in Hamilton-Jacobi equation (20) is a real-valued function of real-valued slowness vector $p_{u}$.

However, eigenvalue $G=G\left(x^{m}, p_{u}\right)$ in Hamilton-Jacobi equation (20) may be a complex-valued function and may generate complex-valued rays. Since we usually do not know the values of the frequency-domain stiffness tensor $c^{i j k l}=c^{i j k l}\left(x^{m}, \omega\right)$ at imaginary coordinates $x^{m}$, we can instead trace the reference real-valued rays which are close to the complex-valued rays defined by the equations (21)-(22) of rays, and to calculate travel time $\tau=\tau\left(x^{m}\right)$ by means of the perturbation expansion by Klimeš and Klimeš (2011) along the reference real-valued rays. In this case, the slowness vector $p_{u}=\tau_{, u}$ in definition (29) is also approximated by the perturbation expansion.

\section{PRINCIPAL AND ADDITIONAL AMPLITUDE COMPONENTS}

Analogously to the zero-order vectorial amplitude (19), we define the amplitude components with respect to the three right-hand eigenvectors of Christoffel matrix (12): eigenvector $g_{i}$ corresponding to the selected eigenvalue $G$ and other two eigenvectors $g_{i}^{\perp}$ corresponding to other two eigenvalues $G^{\perp}$. We decompose each vectorial amplitude coefficient $U_{i}^{[n]}$ in series (7) into the principal amplitude component $U^{[n]}$ and two additional amplitude components $U^{\perp[n]}$,

$$
U_{i}^{[n]}=U^{[n]} g_{i}+\sum_{\perp} U^{\perp[n]} g_{i}^{\perp}
$$




\section{Kliměs}

where the summation is performed over two superscripts ${ }^{\perp}$. Considering expression (19), we assume that both $U^{\perp[0]}=0$.

Definition (9) with decomposition (30) yields

$$
N^{i}\left(U_{m}^{[n]}, \tau_{, u}\right)=\varrho \sum_{\perp} U^{\perp[n]}\left(G^{\perp}-1\right) g_{i}^{\perp}
$$

We multiply viscoelastodynamic equation (14) by left-hand eigenvector $\vec{g}_{i}^{\perp}$ of the Christoffel matrix, consider relation (31), and obtain expression

$$
U^{\perp[n]}=-\varrho^{-1}\left[\vec{g}_{i}^{\perp} M^{i}\left(U_{k}^{[n-1]}, \tau_{, u}\right)+\vec{g}_{i}^{\perp} L^{i}\left(U_{k}^{[n-2]}\right)\right]\left(G^{\perp}-1\right)^{-1}
$$

for the additional amplitude components in terms of lower-order amplitudes. Expression (32) differs from the analogous expression for the symmetric stiffness matrix (Červený, 2001, Eq. 5.7.13) just by left-hand eigenvectors $\vec{g}_{i}^{\perp}$.

\section{TRANSPORT EQUATION}

We multiply viscoelastodynamic equation (14) by left-hand eigenvector $\vec{g}_{i}$ of the Christoffel matrix, consider relation (31), and obtain transport equation

$$
\vec{g}_{i} M^{i}\left(U_{k}^{[n]}, \tau_{, u}\right)+\vec{g}_{i} L^{i}\left(U_{k}^{[n-1]}\right)=0
$$

for the principal amplitude components. We separate the terms with higher-order principal amplitude components from the terms containing higher-order additional amplitude components and lower-order amplitude components,

$$
\vec{g}_{i} M^{i}\left(U^{[n]} g_{k}, \tau_{, l}\right)=-\sum_{\perp} \vec{g}_{i} M^{i}\left(U^{\perp[n]} g_{k}^{\perp}, \tau_{, l}\right)-\vec{g}_{i} L^{i}\left(U_{k}^{[n-1]}\right) .
$$

Relations (33) and (34) differ from the analogous relations for the symmetric stiffness matrix (Červený, 2001, Eqs 5.7.19 and 5.7.20) just by left-hand eigenvector $\vec{g}_{i}$.

We express the left-hand side of transport equation (34) as

$$
\vec{g}_{i} M^{i}\left(U^{[n]} g_{m}, \tau_{, l}\right)=2 \varrho V^{j} U_{, j}^{[n]}+\left(\varrho V^{j}\right),{ }_{j} U^{[n]}-2 \varrho S U^{[n]},
$$

where ray-velocity vector $V^{j}$ is given by definition (29), which can also be expressed as

$$
\varrho\left(x^{a}\right) V^{i}\left(x^{m}, p_{u}\right)=\frac{1}{2} \vec{g}_{r}\left(x^{m}, p_{u}\right)\left[c^{r i k s}\left(x^{a}\right)+c^{r k i s}\left(x^{a}\right)\right] p_{k} g_{s}\left(x^{c}, p_{d}\right) \quad .
$$

The first two terms on the right-hand side of relation (35) are well known from the ray series with a symmetric stiffness matrix (Červený, 2001, Eq. 5.7.23). Quantity $S$ in the rightmost term of transport equation (35) can be determined using definition (10) as

$$
S=\frac{1}{2 \varrho}\left\{\frac{1}{2}\left[\vec{g}_{i}\left(c^{i j k l}+c^{i k j l}\right) \tau_{, k} g_{l}\right]_{, j}-\vec{g}_{i}\left(c^{i j k l} \tau_{, k} g_{l}\right)_{, j}-\vec{g}_{i} c^{i k j l} \tau_{, k} g_{l, j}\right\},
$$

see (36). Considering (35), we express transport equation (34) as

$$
\sqrt{\varrho} V^{j} U_{, j}^{[n]}+\frac{1}{2 \sqrt{\varrho}}\left(\varrho V^{j}\right),{ }_{j} U^{[n]}=\sqrt{\varrho} S U^{[n]}+Z^{[n-1]},
$$


Frequency-domain ray series for viscoelastic waves with a non-symmetric stiffness matrix

where

$$
Z^{[n-1]}=-\frac{1}{2 \sqrt{\varrho}}\left[\sum_{\perp} \vec{g}_{i} M^{i}\left(U^{\perp[n]} g_{k}^{\perp}, \tau_{, u}\right)+\vec{g}_{i} L^{i}\left(U_{k}^{[n-1]}\right)\right] .
$$

The solution of transport equation (38) for $n=0$ reads

$$
U^{[0]}=U_{0}^{[0]}\left(\varrho_{0} J_{0}\right)^{\frac{1}{2}}(\varrho J)^{-\frac{1}{2}} \exp \left(\int_{\tau_{0}}^{\tau} \mathrm{d} \gamma S\right),
$$

where subscript ${ }_{0}$ denotes the initial conditions. Squared geometrical spreading

$$
J=\operatorname{det}\left(\frac{\partial x^{i}}{\partial \gamma^{a}}\right)
$$

(Babich, 1961, Eq. 3.7; Červený, 2001, Eq. 3.10.9) represents the Jacobian of transformation from ray coordinates $\gamma^{1}, \gamma^{2}, \gamma^{3}$ to spatial coordinates $x^{i}$. Here $\gamma^{1}$ and $\gamma^{2}$ are the ray parameters, and $\gamma^{3}=\gamma$.

Factor $\exp \left(\int_{\tau_{0}}^{\tau} \mathrm{d} \gamma S\right)$ in (40) is present due to the skew part of the stiffness matrix, see the next section.

The solution of transport equation (38) for $n>0$ reads (Červený, 2001, Eq. 5.7.30)

$$
U^{[n]}=U^{[0]}\left[\frac{U_{0}^{[n]}}{U_{0}^{[0]}}+\int_{\tau_{0}}^{\tau} \mathrm{d} \gamma \frac{Z^{[n-1]}}{U^{[0]} \sqrt{\varrho}}\right] .
$$

\section{7. "NON-RECIPROCITY"}

\section{DUE TO A NON-SYMMETRIC STIFFNESS MATRIX}

The only difference of expressions (40) and (42) for the principal amplitudes from the analogous expressions derived for a symmetric stiffness matrix is exponential term $\exp \left(\int_{\tau_{0}}^{\tau} \mathrm{d} \gamma S\right)$ in expression (40). We shall now derive various expressions for quantity $S=S\left(x^{m}\right)$.

We express definition (37) as

$$
S=\frac{1}{2 \varrho}\left\{\frac{1}{2}\left[\vec{g}_{i}\left(c^{i j k l}+c^{i k j l}\right) \tau_{, k} g_{l}\right]_{, j}-\left(\vec{g}_{i} c^{i j k l} \tau_{, k} g_{l}\right)_{, j}+\vec{g}_{i, j} c^{i j k l} \tau_{, k} g_{l}-\vec{g}_{i} c^{i k j l} \tau_{, k} g_{l, j}\right\} \text {. }
$$

After summation, we obtain expression

$$
S=\frac{1}{2}\left[\vec{g}_{i, j} a^{i j k l} \tau_{, k} g_{l}-\vec{g}_{i} a^{i k j l} \tau_{, k} g_{l, j}\right]-\frac{1}{4 \varrho}\left[\varrho \vec{g}_{i}\left(a^{i j k l}-a^{i k j l}\right) \tau_{, k} g_{l}\right]_{, j},
$$

where $a^{i k j l}$ is the density-reduced stiffness tensor given by definition (13). The last term on the right-hand side differs from

$$
\frac{1}{2 \varrho}\left(\varrho V^{j}\right)_{, j}=\frac{1}{4 \varrho}\left[\varrho \vec{g}_{i}\left(a^{i j k l}+a^{i k j l}\right) \tau_{, k} g_{l}\right]_{, j}
$$




\section{Kliměs}

just by the subtraction. Note also that

$$
\left[\varrho\left(a^{i j k l}-a^{i k j l}\right) \tau_{, k}\right] \tau_{, j}=0
$$

We differentiate the product in the rightmost term of expression (44) and obtain expression

$S=\frac{1}{4} \vec{g}_{i, j}\left(a^{i j k l}+a^{i k j l}\right) \tau_{, k} g_{l}-\frac{1}{4} \vec{g}_{i}\left(a^{i j k l}+a^{i k j l}\right) \tau_{, k} g_{l, j}-\frac{1}{4 \varrho} \vec{g}_{i}\left[\varrho\left(a^{i j k l}-a^{i k j l}\right)\right]_{, j} \tau_{, k} g_{l}$.

We differentiate characteristic equation (16) for the right-hand eigenvector with respect to spatial coordinates, consider the biorthonormality of the left-hand and right-hand eigenvectors, and obtain relation

$$
g_{i, j}=\sum_{\perp} g_{i}^{\perp} \vec{g}_{k}^{\perp} \Gamma_{, j}^{k l} g_{l}\left(G-G^{\perp}\right)^{-1}+g_{i} \vec{g}_{k} g_{k, j}
$$

for the spatial gradient of the right-hand eigenvector. The rightmost term in relation (48) accounts for the undefined changes of the length of the right-hand eigenvector $g_{i}$. We differentiate characteristic equation (17) for the left-hand eigenvector with respect to spatial coordinates, and obtain analogous relation

$$
\vec{g}_{i, j}=\sum_{\perp} \vec{g}_{k} \Gamma_{, j}^{k l} g_{l}^{\perp} \vec{g}_{i}^{\perp}\left(G-G^{\perp}\right)^{-1}+\vec{g}_{i} g_{k} \vec{g}_{k, j}
$$

for the spatial gradient of the left-hand eigenvector. The rightmost term in relation (49) accounts for the undefined changes of the length of the left-hand eigenvector $\vec{g}_{i}$, and satisfies identity

$$
g_{k} \vec{g}_{k, j}=-\vec{g}_{k} g_{k, j}
$$

obtained by differentiating normalization condition (18).

We insert the gradients (48) and (49) of the eigenvectors of the Christoffel matrix into expression (47), consider identity (50) and arrive at expression

$$
\begin{aligned}
S & =\frac{1}{4} \sum_{\perp}\left(\vec{g}_{k} \Gamma_{, j}^{k l} g_{l}^{\perp} \vec{g}_{r}^{\perp} \frac{\partial \Gamma^{r s}}{\partial p_{j}} g_{s}-\vec{g}_{r} \frac{\partial \Gamma^{r s}}{\partial p_{j}} g_{s}^{\perp} \vec{g}_{k}^{\perp} \Gamma_{, j}^{k l} g_{l}\right)\left(G-G^{\perp}\right)^{-1} \\
& -\frac{1}{4} \vec{g}_{i}\left(c^{i j k l}-c^{i k j l}\right)_{, j} \tau_{, k} g_{l}-\vec{g}_{k} g_{k, j} V^{j},
\end{aligned}
$$

where ray-velocity vector $V^{j}$ is given by definition (29).

We now express the spatial derivatives $\Gamma_{, j}^{k l}$ of the Christoffel matrix in terms of its phase--space derivatives as

$$
\Gamma_{, j}^{k l}=\frac{\partial \Gamma^{k l}}{\partial x^{j}}+\frac{\partial \Gamma^{k l}}{\partial p_{s}} \tau_{, s j} .
$$

The partial derivatives of the Christoffel matrix (12) with respect to phase-space coordinates $x^{m}$ and $p_{u}$ read

$$
\frac{\partial \Gamma^{k l}}{\partial x^{j}}\left(x^{m}, \tau_{, u}\right)=a_{, j}^{k r s l} \tau_{, r} \tau_{, r}
$$


Frequency-domain ray series for viscoelastic waves with a non-symmetric stiffness matrix

and

$$
\frac{\partial \Gamma^{k l}}{\partial p_{j}}\left(x^{m}, \tau_{, u}\right)=\left(a^{k j r l}+a^{k r j l}\right) \tau_{, r} .
$$

Quantity (51) with identity (52) finally reads

$$
\begin{aligned}
S & =\frac{1}{4} \sum_{\perp}\left(\vec{g}_{k} \frac{\partial \Gamma^{k l}}{\partial x^{j}} g_{l}^{\perp} \vec{g}_{r}^{\perp} \frac{\partial \Gamma^{r s}}{\partial p_{j}} g_{s}-\vec{g}_{k} \frac{\partial \Gamma^{k l}}{\partial p_{j}} g_{l}^{\perp} \vec{g}_{r}^{\perp} \frac{\partial \Gamma^{r s}}{\partial x^{j}} g_{s}\right)\left(G-G^{\perp}\right)^{-1} \\
& -\frac{1}{4 \varrho} \vec{g}_{i}\left(c^{i j k l}-c^{i k j l}\right)_{, j} \tau_{, k} g_{l}-\vec{g}_{i} \frac{\mathrm{d} g_{i}}{\mathrm{~d} \gamma} .
\end{aligned}
$$

The last term $\vec{g}_{i} \frac{\mathrm{d} g_{i}}{\mathrm{~d} \gamma}$ in expression (55) represents just the correction of principal amplitude $U^{[n]}$ in decomposition (30) due to the undefined length of right-hand eigenvector $g_{i}$, and vanish if we put

$$
\vec{g}_{i} \frac{\mathrm{d} g_{i}}{\mathrm{~d} \gamma}=0
$$

along each ray.

Each element of matrix $\left(c^{i j k l}-c^{i k j l}\right){ }_{, j} \tau_{, k}$ in the last but one term of relation (55) represents the divergence of a vector tangent to the wavefront, see identity (46). Note also that

$$
\vec{g}_{k} \frac{\partial \Gamma^{k l}}{\partial p_{j}} g_{l}^{\perp} \tau_{, j}=0
$$

and

$$
\vec{g}_{k}^{\perp} \frac{\partial \Gamma^{k l}}{\partial p_{j}} g_{l} \tau_{, j}=0
$$

Vectors

$$
\vec{g}_{k} \frac{\partial \Gamma^{k l}}{\partial p_{j}} g_{l}^{\perp}
$$

and

$$
\vec{g}_{k}^{\perp} \frac{\partial \Gamma^{k l}}{\partial p_{j}} g_{l}
$$

thus represent two sets of the contravariant basis vectors of the ray-centred coordinate system.

Expression (55) for quantity $S$ may be singular at slowness-surface singularities, but is regular at spatial caustics.

Quantity $S$ vanishes for a symmetric stiffness matrix. For a non-symmetric stiffness matrix, quantity $S$ vanishes in a homogeneous medium.

Quantity $S$ which expresses the difference between the ray series for symmetric and non-symmetric stiffness matrices is thus generated by a combination of a non-symmetric stiffness matrix and heterogeneities, and is generally non-zero in a heterogeneous medium with the non-symmetric stiffness matrix. Expression (55) with identities (46), (57) and (58) suggest that quantity $S$ may mostly be influenced by the wavefront-tangent component of the gradient of the skew part of the stiffness matrix. 


\section{Klimeř}

\section{CONCLUSIONS}

We have derived the anisotropic-ray-theory series for viscoelastic waves with a non-symmetric stiffness matrix. These ray series enable us to estimate which phenomena could be observed in the wave field if the stiffness matrix were nonsymmetric.

Whereas the two $\mathrm{S}$ waves, which propagate with different velocities, are linearly polarized in elastic media, they may be elliptically or even circularly polarized in viscoelastic media. Whereas the two elliptically polarized $\mathrm{S}$ waves always display equal handedness for a symmetric stiffness matrix, they display opposite handedness for a sufficiently non-symmetric stiffness matrix, similarly as electromagnetic waves in optically active media.

The ray-theory amplitudes corresponding to a non-symmetric stiffness matrix are not reciprocal in the same way as the ray-theory amplitudes corresponding to a symmetric stiffness matrix. This "non-reciprocity" is expressed in terms of quantity (55) in the expression (40) for the zero-order ray-theory amplitude. Refer to Klimeš (2017, Eq. 18) for the sense in which the ray-theory Green function corresponding to a non-symmetric stiffness matrix is reciprocal.

Acknowledgements: The suggestions by Einar Iversen, Edward S. Krebes and two anonymous reviewers made it possible for me to improve the paper considerably. The research has been supported by the Grant Agency of the Czech Republic under contract 16-05237S, and by the members of the consortium "Seismic Waves in Complex 3-D Structures" (see "http://sw3d.cz").

\section{References}

Babich V.M., 1961. Ray method of calculating the intensity of wavefronts in the case of a heterogeneous, anisotropic, elastic medium. In: Petrashen G.I. (Ed.), Problems of the Dynamic Theory of Propagation of Seismic Waves, Vol. 5. Leningrad Univ. Press, Leningrad, 36-46 (in Russian, English translation: Geophys. J. Int., 118(1994), 379-383).

Červený V., 1972. Seismic rays and ray intensities in inhomogeneous anisotropic media. Geophys. J. R. Astr. Soc., 29, 1-13.

Červený V., 2001. Seismic Ray Theory. Cambridge University Press, Cambridge, U.K.

Červený V., Klimeš L. and Pšenčík I., 2007. Seismic ray method: Recent developments. Adv. Geophys., 48, 1-126.

Chapman C.H., 2004. Fundamentals of Seismic Wave Propagation. Cambridge University Press, Cambridge, U.K.

Hamilton W.R., 1837. Third supplement to an essay on the theory of systems of rays. Trans. Roy. Irish Acad., 17, 1-144.

Klimeš L., 2002. Second-order and higher-order perturbations of travel time in isotropic and anisotropic media. Stud. Geophys. Geod., 46, 213-248. 
Frequency-domain ray series for viscoelastic waves with a non-symmetric stiffness matrix

Klimeš L., 2010. Transformation of spatial and perturbation derivatives of travel time at a general interface between two general media. Seismic Waves in Complex 3-D Structures, 20, 103-114 (http://sw3d.cz).

Klimeš L., 2013. Coupling ray series. Stud. Geophys. Geod., 57, 253-266.

Klimeš L., 2016. Transformation of spatial and perturbation derivatives of travel time at a curved interface between two arbitrary media. Stud. Geophys. Geod., 60, 451-470.

Klimeš L., 2017. Representation theorem for viscoelastic waves with a non-symmetric stiffness matrix. Seismic Waves in Complex 3-D Structures, 27, 93-96 (http://sw3d.cz).

Klimeš M. and Klimeš L., 2011. Perturbation expansions of complex-valued traveltime along real-valued reference rays. Geophys. J. Int., 186, 751-759.

Thomson C.J., 1997. Complex rays and wave packets for decaying signals in inhomogeneous, anisotropic and anelastic media. Stud. Geophys. Geod., 41, 345-381. 